\title{
Persistence of viral RNA in stool samples from patients recovering from covid-19
}

In this editorial by Barth and De Regt (BMJ 2020;369:m1724, doi:10.1136/bmj.m1724, published 7 May 2020), the final sentence in the second paragraph should read: "At the end of these four weeks more than half of the patients still tested positive for SARS-CoV-2 in stool samples using PCR and over a third of patients in serum samples, findings with worrying implications for disease control.” 